ITP-Budapest 538

KEK-TH-551

\title{
Closing the light gluino window ${ }^{1}$
}

\author{
F. Csikor ${ }^{\dagger}$ (csikor@hal9000.elte.hu) \\ Z. Fodor $\dagger^{\dagger}$ (fodor@theory4.kek.jp) \\ ${ }^{\dagger}$ Inst. Theor. Phys., Eötvös University, Budapest, Hungary \\ ${ }^{\dagger \dagger}$ KEK, Theory Group, 1-1 Oho, Tsukuba 305, Japan
}

\begin{abstract}
The running of the strong coupling constant, $R_{e^{+} e^{-}}, R_{Z}$ and $R_{\tau}$ is studied on the three-loop level. Based on experimental data of $R_{e^{+} e^{-}}, R_{Z}$ and $R_{\tau}$ and the LEP multijet analysis, the light gluino scenario is excluded to $99.97 \%$ CL (window I) and $99.89 \%$ CL (window III).
\end{abstract}

\section{Introduction and motivation}

Asymptotic freedom is one of the most interesting predictions of QCD. One can study this by measuring the running coupling constant at different energies and comparing the results. Fig. 1 shows $\alpha_{s}$ running as obtained from different experiments together with QCD predictions. Of course QCD is in very good shape. Nevertheless one may quantify this statement. This is not the subject of the present talk, for details see [1]. One may also speculate on slower running than required by QCD. A popular possibility is the light gluino extension of QCD. In this paper we discuss that this scenario can be excluded using three-loop perturbative results and the existing experimental data [1].

The experimentally excluded regions of light gluinos (1996 status) - mass v. lifetime - are shown in Fig. 2. The moral is that window I., i.e. lighter than 1.5 GeV and window III. i.e. masses between $3 \mathrm{GeV}$ and $5 \mathrm{GeV}$ are allowed by these results.

Last year has brought important technical development of the subject, namely 3-loop results have been calculated [2]-[沺. It has been emphasized that light gluinos are obtained naturally from gauge mediated SUSY breaking [5. 6]. Also some new papers both experimental [7-[9] and theoretical 10, 11] presenting results on light gluino exclusion have appeared. [12]-114 critisizes the exclusion results.

\footnotetext{
* On leave from Inst. Theor. Phys., Eötvös University

${ }^{1}$ Presented by F. Csikor at the International Europhysics Conference on High Energy Physics, Jerusalem, August 19-26 1997.
} 


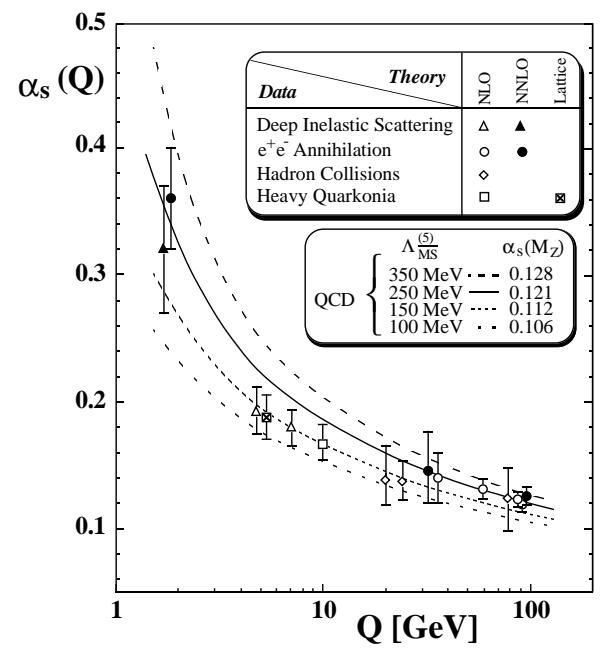

Fig. 1. $\alpha_{s}$ running and QCD predictions

\section{Theoretical and experimental inputs}

Describing $R_{e^{+} e^{-}}, R_{Z}$ and $R_{\tau}$ in the light gluino scenario qualitative differences compared to ordinary QCD appear. Since the effective number of fermions is larger, running of $\alpha_{s}(Q)$ becomes slower. Including the new degrees of freedom in cross section calculations and taking into account the larger phase-space one finds that a smaller $\alpha_{s}$ describes the data at any given energy.

The basic idea of the present paper is the following. We suppose that strong interaction is described by a gauge theory based on a simple Lie-group, thus hadronic cross-sections, widths, $\beta$-function are given by $C_{F}, C_{A}, T_{F}$ and the active number of fermions. Then by looking for $C_{F}, C_{A}, T_{F}, n_{\tilde{g}}$ to describe data accurately, we can determine the CL a given gauge theory is excluded or supported by data. Since the energy range from $M_{\tau}$ to $M_{Z}$ is rather large, it is nontrivial for a hypothetical theory to reproduce both the cross section values and the running of $\alpha_{s}(Q)$.

The theoretical inputs of our analyses are $\mathcal{O}\left(\alpha_{s}^{3}\right)$ calculations of $R_{e^{+} e^{-}}, R_{Z}$ and $R_{\tau}$ for arbitrary group theoretical coefficients. The experimental inputs of the analysis are $R_{\tau}$ given by ALEPH and CLEO $\left.R_{\tau}=3.616(20)\right), R_{Z}$ given by the LEP groups $\left(R_{Z}=20.778(29)\right)$ and hadronic cross sections at energies $5 \mathrm{GeV}-M_{Z}$ (all existing published data (and some unpublished)). The total number of data points included is 182 . 


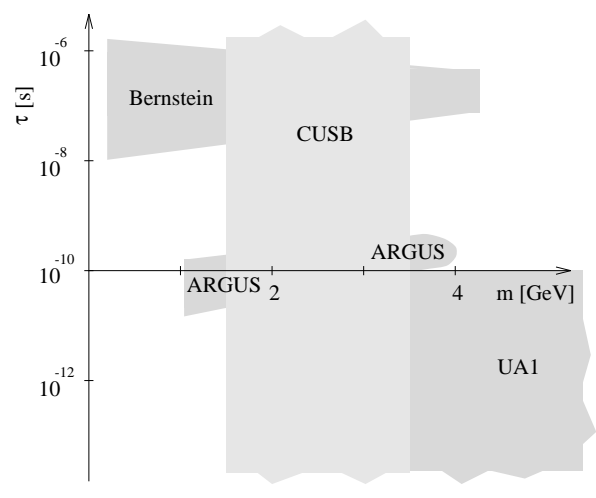

Fig. 2. Light gluino exclusion limits

\section{Treatment of different sources of errors}

Besides the data the errors are also very important for a fit. Being too optomistic destroys reliability of the results. When realized that data imply gluino exclusion, we have choosen to use very conservative error estimates.

We treat uncertainties in a unified manner, add the systematic errors linearly and total systematic and statistical errors quadratically. The accuracy of $R_{e^{+} e^{-}}$and $R_{Z}$ is limited by experiments, while for $R_{\tau}$ theoretical uncertainty dominates. For higher order perturbative corrections we suppose that the error is the last computed term (asymptotic series). For $R_{\tau}$ this gives significantly larger error than usually assumed. For $R_{\tau}$ mass and nonperturbative corrections are taken into account following [15].

Since experimental errors are correlated, we minimize

$$
\chi^{2}=\Delta^{T} V^{-1} \Delta
$$

$\Delta$ is an $n$-vector of the residuals of $R_{i}-R_{f i t}, n$ is the number of individual results, $\mathrm{V}$ is $n \times n$ error matrix.

\section{Light gluino results from running of $\boldsymbol{\alpha}_{s}(Q)$}

First we present results from fiting $R_{e^{+} e^{-}}, R_{Z}, R_{\tau}$, i.e. $\alpha_{s}$ running. An example of a two parameter $\left(x=C_{A} / C_{F}, y=T_{f} / C_{F}\right)$ fit is shown in Fig. 3 for the case of window III. More quantitatively: we exclude window III light gluinos with a small mass dependence to $93(91) \%$ CL for $m_{\tilde{g}}=3(5) \mathrm{GeV}$. For window I we do not use $R_{\tau}$ in the fit and get only $71 \%$ exclusion CL. 


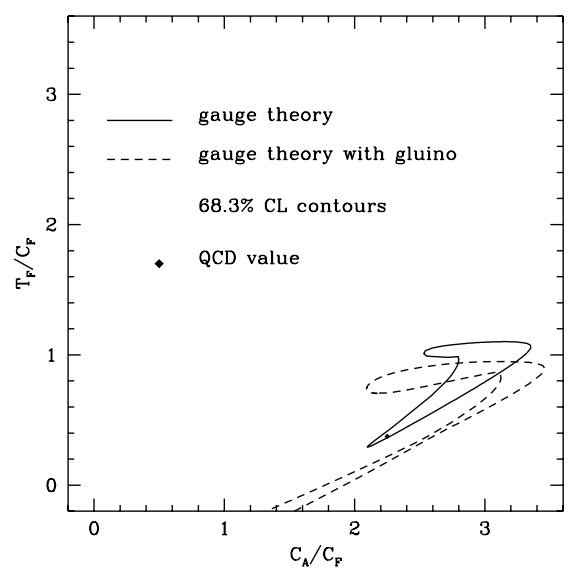

Fig. 3. Exclusion from $\alpha_{s}$ running for $m_{\tilde{g}}=3 \mathrm{GeV}$ (window III)

Following 10 we fixed the underlying group to $\mathrm{SU}(3)$ and determined the number of gluinos. This corresponds to a one parameter fit for $n_{\tilde{g}}$. For window III we get $n_{\tilde{g}}=0.0078 \pm 0.52,88 \%$ CL exclusion and for window I $n_{\tilde{g}}=-0.070 \pm 0.7,80 \% \mathrm{CL}$ exclusion (without $R_{\tau}$ ).

Farrar [12] claims that increasing our $R_{\tau}$ error estimate by a factor 2 leads to $68 \%$ exlusion for window III case. Performing the calculation we get $87(85) \%$ and $81 \%$ for window III 2 and 1 parameter fits, respectively.

\section{Combination with jet analysis at LEP}

LEP groups have determined the group theoretical factors $\mathrm{x}, \mathrm{y}$ comparing experimental results with leading order 4 jet and higher order 2,3 jet predictions. A summary of the earlier results was given in [16] and shown in Fig. 4. To illustrate what happens when our analysis based on $\alpha_{s}$ running is combined with a LEP jet analysis, Fig. 5 shows the $1 \sigma$ excluded regions for the OPAL jet analysis alone [17] (which actually does not exclude light gluinos) and ours (copied from Fig. 3). Note that the overlap of the two regions is rather small, resulting in stronger exclusion than any of the uncombined analyses. It is also clear that increasing the jet analysis ellipsis or shifting it does not change the overlap region too much, i.e. the exclusion changes only very little.

In the actual calculation we have included the older LEP jet analyses and the new ALEPH result [8]. To take into account higher order corrections of 4 jet QCD predictions we have increased the axes of the error ellipse by $12 \%$ of the theoretical $\mathrm{x}$ and y values (relative correction of $\mathcal{O}\left(\alpha_{s}\right)$ ). This change 


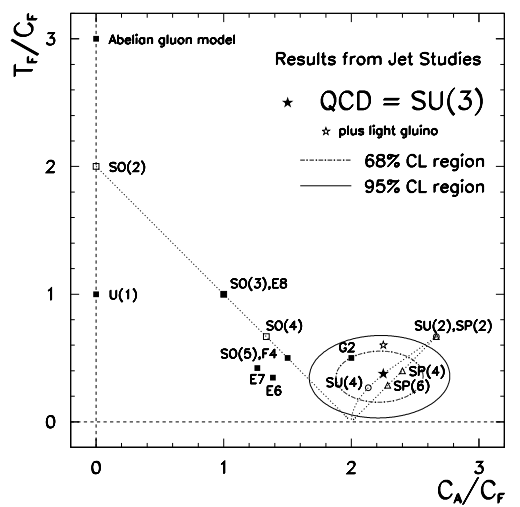

Fig. 4. LEP 4 jet analyses exclusion results

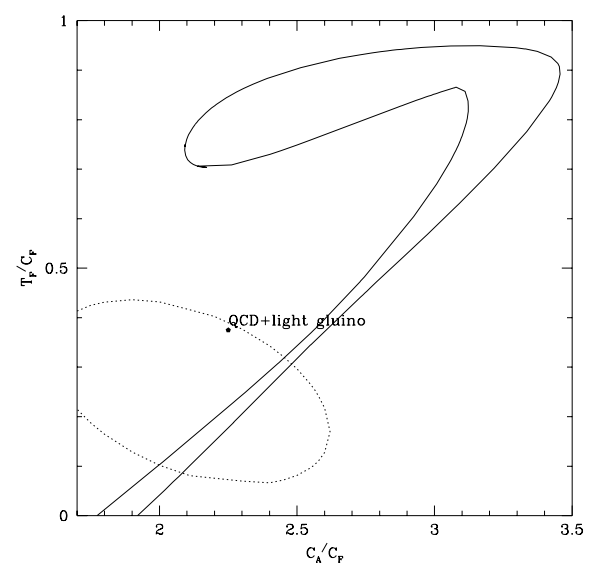

Fig. 5. Comparison of excluded regions from $\alpha_{s}$ running and OPAL 4 jet analysis

would destroy the predictive power of the (uncombined) multi-jet analysis for old data.

An example of the 2 parameter fits is shown in Fig. 6. More quantitatively, for window III light gluino $\left(m_{\tilde{g}}=3(5) \mathrm{GeV}\right)$ the exclusion is $99.99(99.89) \%$ CL. For window I light gluino it is: $99.97 \%$ CL.

We also performed 1 parameter fits. For window III: $n_{\tilde{g}}=-0.156 \pm 0.27$ ($0.197 \pm 0.32$ ) with $m_{\tilde{g}}=3(5) \mathrm{GeV}$ i.e. $99.96(99.76) \%$ CL exclusion. For window I: $n_{\tilde{g}}=-0.35 \pm 0.33$ i.e. we have $99.96 \%$ CL exclusion. 


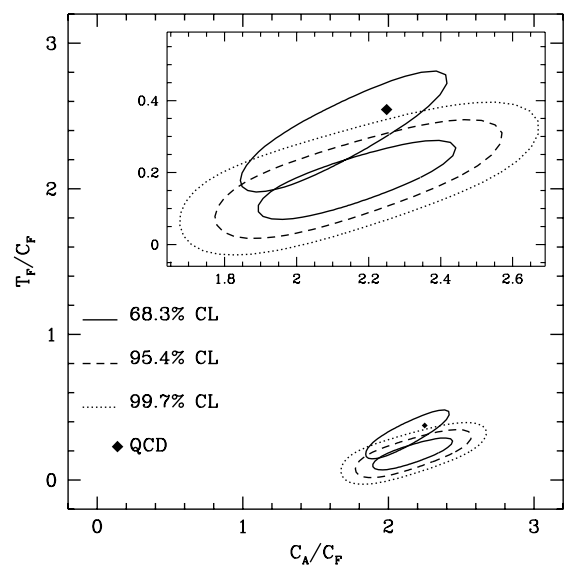

Fig. 6. Exclusion from combined method, 2 parameter fit

Farrar 12 claims that increasing the ALEPH errors by a factor 3 would lead to $68 \%$ gluino exclusion. Performing the actual calculation we get at least $95 \%$ exclusion for all cases of the combined analysis.

\section{Summary, conclusion}

We have obtained light gluino exclusion from fits of $R_{e^{+} e^{-}}, R_{Z}$ and $R_{\tau}$, i.e. essentally from $\alpha_{s}$ running. We have combined this analysis with the LEP jet analysis. Our combined analysis results in much more stringent light gluino exclusion than LEP jet analysis alone or $\alpha_{s}$ running alone. The best CL's are $99.97 \%$ for window I and $99.89 \%$ for window III light gluino exclusion. Our results are independent of light gluino lifetime.

This work was partially supported by Hung. Sci. grants OTKA-T16248,T22929.

\section{References}

[1] F.Csikor, Z.Fodor, Phys. Rev. Letters 78 (1997) 4335 and to be published

[2] K.G. Chetyrkin, Phys. Lett. B391 (1997) 402

[3] L.J. Clavelli, L.R. Surguladze, Phys. Rev. Lett. 78 (1997) 1632

[4] L.J. Clavelli, P.W. Coulter, L.R. Surguladze, Phys. Rev. D55 (1997) 4268

[5] S. Raby, Phys. Rev. D56 (1997) 2852 and this proceedings

[6] R. N. Mohapatra, S. Nandi, Phys. Rev. Letters 79 (1997) 181

[7] F. Abe et al. (CDF), Phys. Rev. D56 (1997) 1357

[8] R. Barate et al., Zeit. Phys. C76 (1997) 191 
[9] J. Adams et al. (KTeV), hep-ex/9709028

[10] A. de Gouvêa, H. Murayama, Phys. Lett. B400 (1997) 117

[11] Z. Nagy, Z. Trócsányi, hep-ph/9708343

[12] G. Farrar, hep-ph/9707467

[13] G. Farrar, hep-ph/9710277

[14] G. Farrar, hep-ph/9710395

[15] G. Altarelli, P. Nason, G. Ridolfi, Z. Phys. C68 (1995) 257

[16] M. Schmelling, , in Proc. of 15th Int. Conf. Physics in Collision, Ed. Frontieres, Gif-Sur-Yvette, p. 287 (1995)

[17] R. Akers et al. (OPAL), Z. Physik C65 (1995) 367 